\title{
Thrust Enhanced by a Magnetic Laval Nozzle in an Applied-Field Magneto-Plasma-Dynamic Thruster ${ }^{*)}$
}

\author{
Hiroaki NABUCHI, Kiyotaka SUZUKI, Yohei KOBAYASHI, Atsushi KOMURO, \\ Kazunori TAKAHASHI and Akira ANDO \\ Department of Electrical Engineering, School of Engineering, Tohoku University, \\ 6-6-05 Aoba-yama, Sendai 980-8579, Japan
}

(Received 30 November 2015 / Accepted 1 February 2016)

\begin{abstract}
The thrust imparted by an applied-field magneto-plasma-dynamic thruster is enhanced by superimposing a convergent-divergent magnetic field called a magnetic Laval nozzle. The thrust increases up to $6.9 \mathrm{~N}$ by increasing the magnetic field strength at the Laval nozzle throat. It is observed that the plasma flow velocity increases and the ion temperature simultaneously decreases downstream of the throat. A major component of the measured Lorentz force arising from the plasma-induced current is identified to be in the radial direction, while a significant increase in the plasma density is observed upstream of the nozzle. These results imply that the plasma pressure increases upstream of the magnetic Laval nozzle due to an inhibition of the plasma loss, which contributes to the increase of the thrust.
\end{abstract}

(C) 2016 The Japan Society of Plasma Science and Nuclear Fusion Research

Keywords: Magneto-Plasma-Dynamic (MPD) thruster, magnetic Laval nozzle, high-density plasma, electric propulsion

DOI: $10.1585 /$ pfr.11.2406033

\section{Introduction}

The interaction between plasma and magnetic fields is one of the key issues in developing electric propulsion devices $[1,2]$. Plasma acceleration and thrust generation in magnetic nozzles have been vigorously investigated in various types of electric propulsion concepts, e.g., variable specific impulse magnetoplasma rockets (VASIMR) [3], applied-field magneto-plasma-dynamic (AF-MPD) thrusters [4, 5], and helicon plasma thrusters $[6,7]$. These thrusters operate on a wide range of power from a few $\mathrm{kW}$ for the helicon thruster and a few hundreds of $\mathrm{kW}$ for VASIMR to MW for the MPD thruster.

The AF-MPD thruster has a solenoid, which provides an external magnetic field, in addition to a simple selfinduced MPD thruster comprising a central cathode and an annular anode, where electric current of a few $\mathrm{kA}$ is supplied to the electrodes. Propellant gas is highly ionized and accelerated by the Lorentz force, which arises from the discharge current and self-induced magnetic field [8].

Because the thrust density given by the thrust over the cross section is much higher than that of other propulsion devices, it is a candidate propulsion system for missions such as massive material transport and manned exploration in space. By applying the magnetic nozzle or an external magnetic field to the MPD thruster, various effects originating from the Lorentz force, plasma confinement, and variation in the plasma cross section have been proposed

author'se-mail:nabuchi@ecei.tohoku.ac.jp

*) This article is based on the presentation at the 25th International Toki Conference (ITC25). and experimentally observed [9-13]. Sasoh has formulated hall and swirl acceleration processes in the magnetic nozzle, which have been experimentally validated [14].

Improvements in the thruster performance have also been demonstrated by several researchers using divergent magnetic fields at the MPD electrodes to enhance the thrust and axial flow velocity [15]. However, it has also been observed that the axial flow energy is thermalized downstream of the divergent magnetic field. Such behavior of the plasma flow in a magnetic nozzle can be understood using a simple, one-dimensional, isentropic flow model. The isentropic flow model is equivalent to a two-dimensional magnetic nozzle model assuming a negligible ion temperature in the helicon thruster [16].

Although the magnetic nozzle seems to be equivalent to a physical nozzle, the force arising in a magnetic nozzle, given by the Lorentz force integrated over the volume, accelerates the plasma flow, and the reaction force is simultaneously exerted on the magnetic field lines [11]. On the other hand, the force due to the physical nozzle is a surface force exerted on a physical boundary. Such physical pictures are expected to fit the case of the AF-MPD thruster. In well-known fluid dynamics, given a one-dimensional nozzle model, a Laval nozzle can effectively accelerate fluids when having a subsonic velocity upstream of the nozzle throat.

Here, the Laval nozzle structure is formed by a magnetic field and installed in the AF-MPD thruster; therefore, it is called a magnetic Laval nozzle. The effect of the magnetic Laval nozzle on plasma parameter has been investi- 
gated previously [17]. The thrust imparted by the plasma flow under the magnetic Laval nozzle is measured using the pendulum target technique. The result clearly shows an increase in the thrust when increasing the magnetic field strength of the nozzle throat. Based on the axial flow velocity, ion temperature, electromagnetic force, and ion density measured downstream of the thruster head, mechanisms of the thrust increase is discussed.

\section{Experimental Setup}

The experiments are performed in the HITOP (High density Tohoku Plasma) device at Tohoku University, which can be found in Ref. [17, 18]. The setup comprises an AF-MPD thruster, a vacuum chamber, and magnetic field coils, which generate a uniform magnetic field. The AF-MPD thruster shown in Fig. 1 is presently installed at one end-port of the HITOP device. It has a coaxial structure with a central tungsten rod cathode $(10 \mathrm{~mm}$ in outer diameter) and an annular molybdenum anode $(30 \mathrm{~mm}$ in inner diameter). Two solenoid coils are set near the MPD thruster exit to apply the magnetic diverging nozzle and magnetic Laval nozzle.

Figure 2 shows the calculated axial profiles of the magnetic field strength on the axis. The uniform field, $B_{0}$ $=0.10 \mathrm{~T}$, the diverging magnetic nozzle, $B_{\mathrm{zc}}=0.28 \mathrm{~T}$, and the magnetic Laval nozzle, $B_{\mathrm{zL}}$, corresponding to the magnetic nozzle throat $(Z=100 \mathrm{~mm})$, are superimposed, where

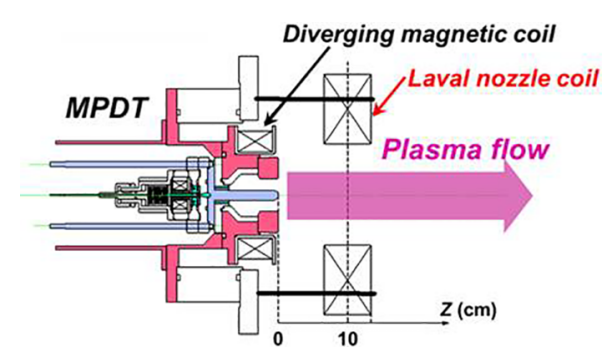

Fig. 1 Schematic of an AF-MPD thruster with two external magnetic coils.

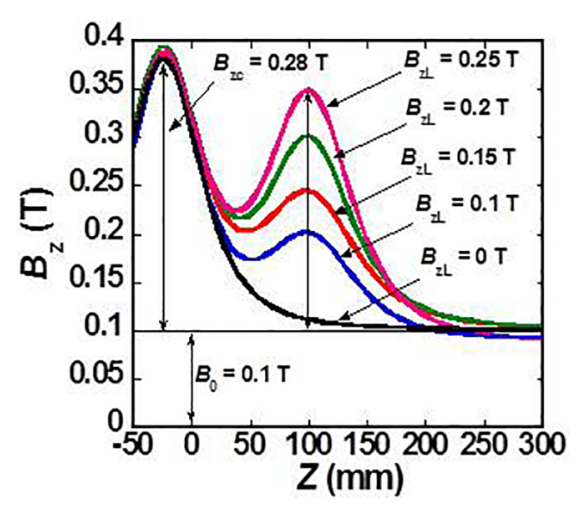

Fig. 2 Calculated axial profiles of the magnetic field strength on the axis for various values of $B_{\mathrm{zL}}$.
$Z=0$ is defined as shown in Fig. 2. The five configurations being tested here are labeled $B_{\mathrm{zL}}=0,0.10,0.15,0.20$, and $0.25 \mathrm{~T}$.

Helium gas is used as a propellant and injected from the upstream side of the thruster via a fast acting valve system with a mass flow rate of $38 \mathrm{mg} / \mathrm{s}$. A discharge current of $I_{\mathrm{d}}=6.8 \mathrm{kA}$ is supplied by a pulse forming network (PFN) system with a duration of approximately $1 \mathrm{~ms}$, where $I_{\mathrm{d}}$ can be controlled by the charging voltage of a capacitor bank in the PFN power supply.

To measure the thrust, a pendulum-type thrust target [19] is installed within the HITOP vacuum chamber, which can be found in Ref. [15]. A detailed description of the procedure for the thrust measurement can be found in Ref. [19]. In brief, the pulsed plasma flow impinges on the target and causes the pendulum to oscillate, which is measured by a commercial laser displacement sensor. The impulse bit $\left(I_{\mathrm{bit}}=F \Delta t\right)$ is evaluated by the amplitude of the first swing and a calibration coefficient relating to the amplitude and the given impulse bit, which is obtained before pumping down the chamber. The thrust is estimated from the impulse bit, $I_{\text {bit }}$, imparted by the plasma flow and discharge pulse width, $\Delta t$.

The plasma flow velocity, $U_{\mathrm{z}}$, and ion temperature, $T_{\mathrm{i}}$, are measured by an optical emission spectroscopy [18]. The optical lens including a collimator and a fiber are mounted on a movable optical stage near the quartz viewing side port located near the MPD thruster exit. The line spectrum emission of the He II line $\left(\lambda_{0}=468.575 \mathrm{~nm}\right)$ is measured from two directions, perpendicular and oblique to $Z$ axis. $U_{\mathrm{z}}$ is evaluated using the Doppler shift, $\Delta \lambda_{\mathrm{D}}$, of the spectrum peaks that are perpendicularly and obliquely observed. $T_{\mathrm{i}}$ is obtained from the broadness of the spectrum line. Even though the measured $T_{\mathrm{i}}$ is the radial ion temperature, i.e., a component perpendicular to the magnetic field, it is assumed to be isotropic in the discussions in this study.

A planner Langmuir probe and three-axis magnetic probes are mounted on a stage movable in the $x-z$ plane to measure the plasma density and magnetic field variation induced by the plasma current.

\section{Experimental Results}

Figure 3 shows the measured thrust as a function of the magnetic field strength, $B_{\mathrm{zL}}$, where the discharge current is kept at $I_{\mathrm{d}}=6.8 \mathrm{kA}$. The thrust increases up to $\sim 6.9 \mathrm{~N}$ with the increase in $B_{\mathrm{ZL}}$ for a field strength of $B_{\mathrm{ZL}}<\sim 0.15$ T. This result demonstrates that the AF-MPD thruster performance can be improved by applying the magnetic Laval nozzle. For $B_{\mathrm{zL}}>0.15 \mathrm{~T}$, the thrust seems to be saturated or to slightly decrease when $B_{\mathrm{zL}}$ is increased further.

To discuss and understand the physical mechanisms of the thrust increase by the magnetic Laval nozzle, axial profiles of the plasma flow velocity, $U_{\mathrm{z}}$, and ion temperature, $T_{\mathrm{i}}$, are measured along the axis, as shown in Figs. 4 (a) 


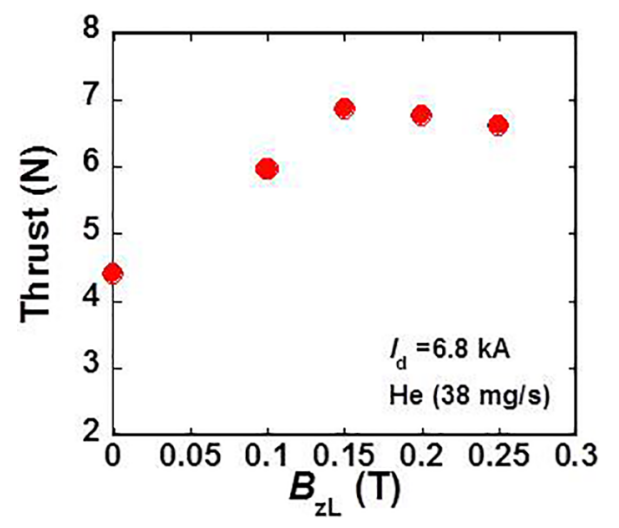

Fig. 3 The measured thrust as a function of the magnetic field strength, $B_{\mathrm{zL}}$, of the Laval nozzle throat for $I_{\mathrm{d}}=6.8 \mathrm{kA}$.

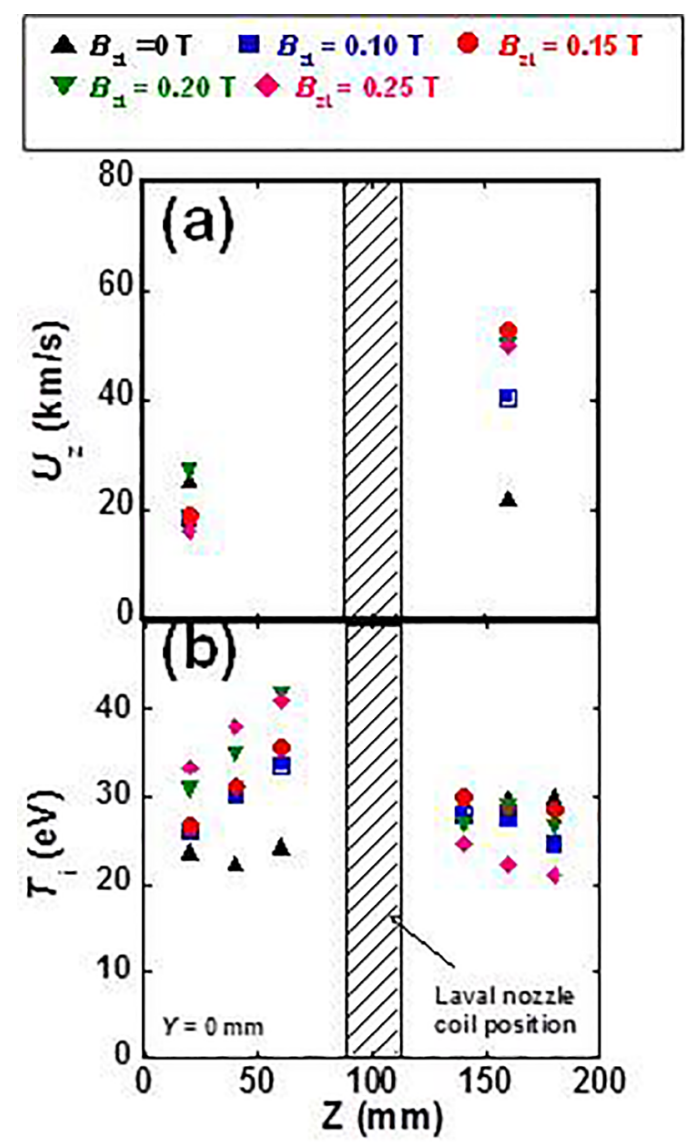

Fig. 4 Axial profiles of (a) the plasma flow velocity, $U_{z}$, and (b) the ion temperature, $T_{\mathrm{i}}$, for $I_{\mathrm{d}}=6.8 \mathrm{kA}$. The shaded region near $Z=100 \mathrm{~mm}$ corresponds to the location of the Laval nozzle coil.

and 4 (b), respectively, for various field strengths $B_{\mathrm{zL}}$. Because the shaded region near $z=100 \mathrm{~mm}$ in Fig. 4 corresponds to the location of the Laval nozzle coil, no optical measurement is performed there due to geometrical problems. For the case with no Laval nozzle, plotted by filled triangles, $U_{\mathrm{z}}$ slightly decreases and $T_{\mathrm{i}}$ increases along the axis. These results are consistent with previous experiments [15]. However, when applying the magnetic Laval
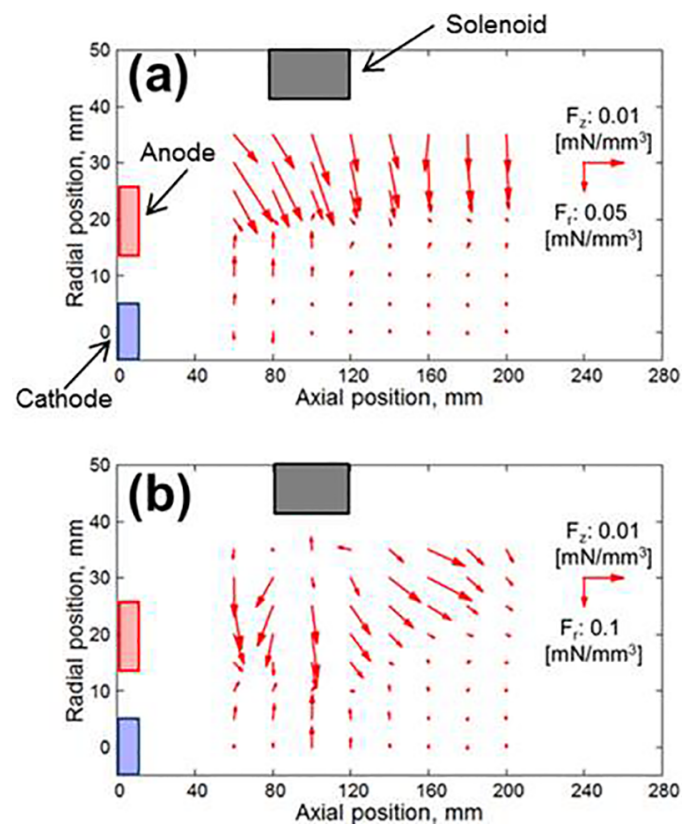

Fig. 5 Two-dimensional vector plots of the $r$ and $z$ components of the Lorentz force, $F_{\mathrm{z}}$ and $F_{\mathrm{r}}$, for (a) $B_{\mathrm{zL}}=0 \mathrm{~T}$ and (b) $B_{\mathrm{zL}}=0.15 \mathrm{~T}$, respectively. The discharge current $I_{\mathrm{d}}$ is maintained at $6.8 \mathrm{kA}$.

nozzle, it is found that $U_{\mathrm{z}}$ dramatically increases and $T_{\mathrm{i}}$ decreases in the downstream region, as seen in Figs. 4 (a) and 4 (b). These results suggest that the ion thermal energy is converted into axially directed flow energy by the presence of the magnetic Laval nozzle.

Axial plasma acceleration can be induced by various effects, such as hydrodynamic and electromagnetic acceleration processes, where the reaction force is exerted on the mechanical and magnetic structures, respectively. Here, the Lorentz force exerted on the magnetic structure is evaluated from the plasma-induced magnetic field, $\Delta B_{\mathrm{z}}, \Delta B_{\mathrm{r}}$, and $\Delta B_{\theta}$, which are measured by the magnetic probes and gives the net current flowing inside the plasma.

Figures 5 (a) and 5 (b) show two-dimensional vector plots of the Lorentz force fields, $F_{\mathrm{z}}$ and $F_{\mathrm{r}}$, in the $r-z$ plane, which are exerted on the plasma flow for $B_{\mathrm{zL}}=0 \mathrm{~T}$ (without a magnetic Laval nozzle) and $B_{\mathrm{zL}}=0.15 \mathrm{~T}$, respectively. A force equal in magnitude and opposite in direction to the forces in Fig. 5 is exerted on the magnetic field structure and generates the thrust. For both cases, with and without the magnetic Laval nozzle, it is found that the radial component of the Lorentz force, $F_{\mathrm{r}}$, is much larger than the axial component, $F_{\mathrm{z}}$, as shown in Figs. 5 (a) and 5 (b). The charging voltage of the PFN circuit is adjusted to keep a constant net current between the cathode and the anode in the various field strength of the magnetic Laval nozzle. The integrated values of $F_{\mathrm{z}}$ over the measurement region are $1.0 \mathrm{~N}$ without and $1.1 \mathrm{~N}$ with the magnetic Laval nozzle. Therefore, the contribution of the plasma acceleration in the magnetic nozzle to the thrust is only $16 \%$ of the measured thrust. 

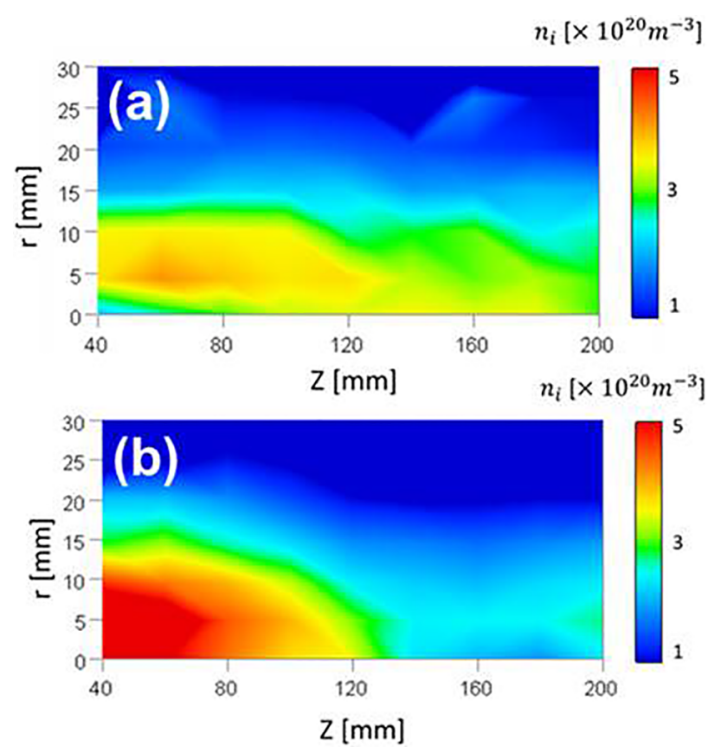

Fig. 6 Two dimensional distributions of the ion density for (a) $B_{\mathrm{zL}}=0 \mathrm{~T}$ and (b) $B_{\mathrm{zL}}=0.15 \mathrm{~T}$, respectively. $I_{\mathrm{d}}$ is reduced to $4.0 \mathrm{kA}$ to avoid thermal damage in the Langmuir probe.

According to the above-mentioned Lorentz force contribution, a major contribution is expected due to a hydrodynamic acceleration process, in which a pressure is converted into fluid dynamic momentum. To verify this scenario, the ion density, $n_{\mathrm{i}}$, is estimated from the ion saturation current of a Langmuir probe with a 0.9 -mm-diameter tungsten tip, where the discharge current is reduced to $I_{\mathrm{d}}$ $=4.0 \mathrm{kA}$ to prevent damage to the Langmuir probe from the high density plasma flow. Figures 6 (a) and 6 (b) show the two-dimensional distributions of the ion density for $B_{\mathrm{zL}}$ $=0 \mathrm{~T}$ (without a magnetic Laval nozzle) and $B_{\mathrm{zL}}=0.15$ $\mathrm{T}$, respectively. It is found that the density at $Z<100$ $\mathrm{mm}$ (upstream of the magnetic Laval nozzle) increases in Fig. 6 (b) compared to Fig. 6 (a). Therefore, the increased plasma pressure upstream of the nozzle throat seems to contribute to the thrust increase by increasing $B_{\mathrm{zL}}$ in Fig. 3 . More interestingly, the density downstream of the nozzle throat significantly decreases in Fig. 6 (b); therefore, a strong pressure gradient exists along the axis. The presence of this pressure gradient leads to axial plasma acceleration, i.e., the increase in $U_{\mathrm{z}}$ in Fig. 4.

\section{Conclusion}

A magnetic Laval nozzle is superimposed on the AFMPD thruster resulting in a divergent magnetic field struc- ture near the thruster exit. It is found that the thrust increases with the application of a magnetic Laval nozzle. The axial flow velocity increases and the ion temperature decreases downstream of the nozzle throat. This suggests that ion thermal energy is converted into directed flow energy through the magnetic Laval nozzle. Two mechanisms of thrust enhancement induced by the magnetic Laval nozzle, i.e., electromagnetic and hydrodynamic acceleration processes, are discussed on the basis of the measured Lorentz force and plasma density. The direct measurement of the Lorentz force shows that the electromagnetic acceleration process has only a $16 \%$ contribution to the thrust increase, while the radial component of the electromagnetic force seems to play an important role in the plasma compression and its resultant confinement in the radial direction. Because plasma loss is inhibited by the radial force, the radially-integrated plasma pressure upstream of the nozzle throat increases and gives larger thrust in the case with the magnetic Laval nozzle.

\section{Acknowledgement}

This work is partially supported by grants-in-aid for scientific research (A26247096 and B 25287150) from the Japan Society for the Promotion of Science and the NIFS Collaboration Research program (NIFS13KBA007).

[1] K. Dannenmayer and S. Mazouffre, J. Propul. Power 27, 236 (2011).

[2] D.M. Goebel and I. Katz, Fundamentals of electric propulsion: ion and hall thrusters (John Wiley \& Sons, Inc., publication 2008).

[3] B.W. Longmier et al., J. Propul. Power 27, 4 (2011).

[4] R.M. Myers, J. Propul. Power 9, 5 (1993).

[5] K. Sankaran et al., J. Propul. Power 21, 1 (2005).

[6] C. Charles, J. Phys. D: Appl. Phys. 42, 163001 (2009).

[7] K. Takahashi et al., Phys. Rev. Lett. 110, 195003 (2013).

[8] I. Funaki et al., J. Propul. Power 14, 6 (1998).

[9] A. Sasoh et al., J. Propul. Power 11, 351 (1995).

[10] A. Fruchutman, Phys. Rev. Lett. 96, 065002 (2006).

[11] K. Takahashi et al., Phys. Rev. Lett. 107, 235001 (2011).

[12] A.V. Areviev and B.N. Breizmann, Phys. Plasmas 12, 043504 (2005).

[13] E. Ahedo and M. Merino, Phys. Plasmas 17, 073501 (2010).

[14] A. Sasoh, Phys. Plasmas 1, 3 (1994).

[15] Y. Izawa et al., JPS Conf. Proc. 1, 015046 (2014).

[16] A. Fruchtman et al., Phys. Plasmas 19, 0333507 (2012).

[17] M. Inutake et al., Plasma Phys. Control. Fusion 49, A121 (2007).

[18] H. Tobari et al., Phys. Plasmas 14, 093507 (2007).

[19] Y. Takao et al., J. Appl. Phys. 101, 123307 (2007). 